\title{
Dipole Scattering Amplitude in Momentum Space: Investigating Fluctuations at HERA
}

\author{
E. Basso, ${ }^{*}$ M. B. Gay Ducati,$^{\dagger}$ E. G. de Oliveira ${ }^{\ddagger}$ and J. T. de Santana Amaral ${ }^{\S}$ \\ Instituto de Física, Universidade Federal do Rio Grande do Sul, \\ Caixa Postal 15051, 91501-970 - Porto Alegre, RS, Brazil
}

(Received on 4 August, 2008)

\begin{abstract}
We extend a recently proposed dipole model which relates the virtual photon-proton cross section to the dipole-proton forward scattering amplitude in momentum space investigating the effects of the gluon number fluctuations. The model interpolates between well known asymptotic behaviours predicted by perturbative QCD from the Balitsky-Kovchegov equation, which describes the rapidity evolution of the dipole-proton scattering amplitude in the mean field approximation. The model was shown to be successful in describing the last HERA data for the case where the strong coupling constant $\alpha_{s}$ is fixed, showing also some important advantages when compared with other dipole models - all of them in coordinate space - in the literature. Based on the fact that the fluctuations may be important in the small- $x$ evolution and on recent results obtained in coordinate space beyond the mean field approximation, we use this model to parametrize the proton structure function and confront it to HERA data using the average (physical) amplitude - then including fluctuations - within the momentum space framework.
\end{abstract}

Keywords: Evolution equations; Dipole picture; Scattering amplitudes; DIS and fluctuations

\section{INTRODUCTION}

The dipole picture [1] is a powerful framework through which it is possible to understand many important features of scattering ane evolution in QCD at high energies. In particular, it allows one to describe the evolution of scattering amplitudes in hadronic interactions. In this picture, a gluon can be replaced by a colorless quark-antiquark pair, a dipole, and a gluon-target scattering becomes a dipole-target scattering. If one considers a projectile made of one dipole, which interacts with a dense target, for example, a proton, the evolution of the dipole-proton scattering amplitude is given, in the limit where the number of colors $N_{c}$ is large, by the so called BalitskyJIMWLK equations [2,3], which consist of an infinite hierarchy which relates the one-dipole scattering amplitude with the two-dipole amplitude, whose evolution depends on the threedipole amplitude and so on.

Of course, to deal with this infinite hierarchy it is not an easy task. However, if one performs a mean field approximation, this set of equations reduces to a single equation which describes the evolution in rapidity $Y$ (the logarithm of the energy) of the one-dipole scattering amplitude $T(, y, Y)$ of a dipole with a quark and the antiquark whose transverse coordinates are $x$ and $y$. This mean field version of BalitskyJIMWLK equations is known as BK (Balitsky-Kovchegov) equation $[2,4]$.

From a pioneering analysis of BK equation and its solutions [5], it became clear that the high energy evolution in QCD is similar to a reaction-diffusion process in statistical physics. From this correspondence, it has been also realized that the gluon number fluctuations are important in the evolution towards high energy (small- $x$ ). These fluctuations are

\footnotetext{
${ }^{*}$ Electronic address: ebasso@if.ufrgs.br

†Electronic address: beatriz.gay@ufrgs.br

†Electronic address: emmanuel. deoliveira@ufrgs.br

$\S$ Electronic address: thiago.amaral@ufrgs.br
}

not included in the original Balitsky-JIMWLK hierarchy and obviously missed in its mean field version, the BK equation.

The fluctuations have been exhaustively investigated in recent years and their effects in the high energy evolution will be discussed in the next sections of this contribution. What one can antecipate is that they dramatically influence the high energy scattering, at least in the case when the strong coupling constant $\alpha_{s}$ is fixed. Among these investigations, one can cite a recent study [6] of the possible implications of the gluon number fluctuations in the measurements of the $F_{2}$ proton structure function of deep inelastic scattering (DIS) at HERA, through well known models for the dipole-proton cross section in coordinate space $[7,8]$. The results show that, although they may be present in the DIS measurements, they seem not to strongly influence the evolution at HERA energies.

In these proceedings we use the first model for the dipoleproton cross section developed in momentum space [9] with fluctuation effects included to fit the last HERA data. This model has shown to be successful in the description of DIS data, specially with heavy quark (charm) contributions included, by using a mean-field (BK) inspired parametrization for the scattering amplitude, which enters in the expression for the dipole-proton cross section. Some preliminary results are presented and some future expectations on the effects of fluctuations at HERA are discussed.

\section{2. $\gamma^{*}-p$ CROSS SECTION IN THE DIPOLE PICTURE}

In the electron-proton deep inelastic scattering (DIS) the projectile and the target interact by exchanging a virtual photon of virtuality $Q^{2}$. At high energies, this process can be seen in two steps: first, the virtual photon split into a quarkantiquark pair, a dipole, which then interacts with the proton. This is the dipole frame in which the virtual photon-proton cross section can be written in a factorized form, a convolution between the (longitudinal and transversal) wavefunctions for the virtual photon to go into the colorless dipole and the 
dipole-proton cross section $\sigma_{\mathrm{dip}}$

$$
\sigma_{T, L}^{\gamma^{*} p}\left(Q^{2}, Y\right)=\int d^{2} r \int_{0}^{1} d z\left|\Psi_{T, L}\left(r, z ; Q^{2}\right)\right|^{2} \sigma_{\mathrm{dip}}(r, Y),
$$

where the rapidity variable $Y$ is defined in terms of the square of the total energy of the process, or the inverse of the Bjorken variable $x$, by $Y \equiv \ln 1 / x=\ln s$ and $r$ is the vector which gives the transverse size of the dipole, composed by a quark and an antiquark which carry a fraction $z$ and $1-z$ of the momentum of the photon, respectively.

From Eq.(1) it is possible to write the $F_{2}$ structure function through the relation

$$
F_{2}\left(x, Q^{2}\right)=\frac{Q^{2}}{4 \pi^{2} \alpha_{e m}}\left[\sigma_{T}^{\gamma^{*} p}\left(x, Q^{2}\right)+\sigma_{L}^{\gamma^{*} p}\left(x, Q^{2}\right)\right] .
$$

From the optical theorem, the dipole-proton cross section, which must be modelled, can be expressed in terms of the dipole-proton scattering amplitude $\langle T(r, b)\rangle_{Y}$ at a given impact parameter $b$, where the average is taken over the target wavefunction. The procedure of modelling $\sigma_{\text {dip }}$ is not new and among the models in the literature one can cite the GBW (Golec-Biernat and Wusthoff) model [7] and the IIM (Iancu, Itakura and Munier) model [8], which is based on perturbative QCD predictions for the scattering amplitudes. Both parametrizations are constructed in the $b$-independent case, for which the dipole-proton cross section can be written as

$$
\sigma_{d i p}^{\gamma^{*} p}(r, Y)=2 \pi R_{p}^{2}\langle T(r, Y)\rangle,
$$

where $R_{p}$ is the radius of the proton. From now on we will omit the brakets and write $T(r, Y)$ only to denote the average amplitude. If one Fourier transforms the amplitude $T(r, Y)$, the dipole-proton cross section can be written in terms of the amplitude in momentum space, $\tilde{T}(k, Y)$. The $F_{2}$ structure function then takes the form [9]

$$
F_{2}\left(x, Q^{2}\right)=\frac{Q^{2} R_{p}^{2} N_{c}}{4 \pi^{2}} \int_{0}^{\infty} \frac{d k}{k} \int_{0}^{1} d z\left|\tilde{\Psi}\left(k^{2}, z ; Q^{2}\right)\right|^{2} \tilde{T}(k, Y),
$$

where the wavefunction is now expressed in momentum space.

\section{THE AGBS MODEL}

All the parametrizations for the scattering amplitude cited above are developed in coordinate space, i.e., provide an expression for $T(r)$. However, in a recent publication, a different parametrization was proposed for the $\sigma_{\text {dip }}$, the AGBS model (Amaral, Gay Ducati, Betemps and Soyez) [9], which is expressed in terms of $\tilde{T}$. This is the first parametrization for the scattering amplitude in momentum space in the literature and will be described in what follows

The AGBS model interpolates between two asymptotic behaviors of the scattering amplitude predicted by perturbative QCD in the framework of the mean-field Balitsky-Kovchegov (BK) nonlinear evolution equation [2,4] (in momentum space, of course). These asymptotic behaviors come from the properties of solutions of BK equation in the saturated (infrared) and dilute regimes, given, respectively, by

$$
\tilde{T}(k, Y) \stackrel{k \ll Q_{s}}{=} c-\log \left(\frac{k}{Q_{s}(Y)}\right)
$$

and

$$
\begin{aligned}
& \tilde{T}(k, Y) \stackrel{k \gg Q_{s}}{\approx}\left(\frac{k^{2}}{Q_{s}^{2}(Y)}\right)^{-\gamma_{c}} \log \left(\frac{k^{2}}{Q_{s}^{2}(Y)}\right) \\
& \times \exp \left[-\frac{\log ^{2}\left(k^{2} / Q_{s}^{2}(Y)\right)}{2 \bar{\alpha} \chi^{\prime \prime}\left(\gamma_{c}\right) Y}\right] .
\end{aligned}
$$

Here $Q_{s}(Y)=\exp (\lambda Y)$ is the so called saturation momentum, and $\lambda$ the saturation exponent, given by

$$
\lambda=\min \bar{\alpha} \frac{\chi(\gamma)}{\gamma}=\bar{\alpha} \frac{\chi\left(\gamma_{c}\right)}{\gamma_{c}}=\bar{\alpha} \chi^{\prime}\left(\gamma_{c}\right) .
$$

where $\bar{\alpha} \equiv \alpha_{s} N_{c} / \pi$ and $\chi(\gamma)$ is the BFKL kernel [10]. For the LO kernel, $\gamma_{c}=0.6275 \ldots$

The interpolation in the AGBS model is done through the following expression for the scattering amplitude

$$
T(k, Y)=\left[\log \left(\frac{k}{Q_{s}}+\frac{Q_{s}}{k}\right)+1\right]\left(1-e^{-T_{\mathrm{dil}}}\right) .
$$

where

$$
\tilde{T}_{\text {dil }}=\exp \left[-\gamma_{c} \log \left(\frac{k^{2}}{Q_{s}^{2}(Y)}\right)-\frac{\mathcal{L}^{2}-\log ^{2}(2)}{2 \bar{\alpha} \chi^{\prime \prime}\left(\gamma_{c}\right) Y}\right]
$$

with

$$
\mathcal{L}=\log \left[1+\frac{k^{2}}{Q_{S}^{2}(Y)}\right] \quad \text { and } \quad Q_{s}^{2}(Y)=k_{0}^{2} e^{\lambda Y} .
$$

\section{PROPERTIES OF THE SCATTERING AMPLITUDES}

BK equation is the most simple nonlinear equation which describes the evolution of the scattering amplitude towards the high energy limit, and some of its properties are well known. In particular, it has been shown [5] that this equation admits traveling waves solutions, which are a natural explanation to the geometric scaling observed in the $\gamma^{*}-p$ cross section at HERA. The scattering amplitude is a front which travels towards higher values of transverse momenta $k$ and, at asymptotic rapidities, depends only on the scaling variable $\ln k^{2} / Q_{s}^{2}$, $\ln Q_{s}^{2}$ being the front position and $\lambda$ its asymptotic velocity.

Although BK equation is very important for the understanding of the high energy evolution in QCD, as it was explained in the Introduction, it is only a mean field description of the dipole evolution, it is the mean field approximation of the first equation of the Balitsky-JIMWLK hierarchy [2,3], which has been recently generalized to include the effects of the gluon number fluctuations, resulting in the Pomeron loop equations [11]. 


\begin{tabular}{c||c|c|c|c|c|c|}
\hline \hline model & $\chi 2 /$ n.o.p & $k_{0} 2\left(\times 10^{-3}\right)$ & $\lambda$ & $R\left(\mathrm{GeV}^{-1}\right)$ & $\chi^{\prime \prime}\left(\gamma_{c}\right)$ & $D$ \\
\hline \hline$\tilde{T}^{\text {AGBS }}$ & 0.960 & $3.782 \pm 0.301$ & $0.213 \pm 0.003$ & $3.576 \pm 0.059$ & $4.6903 \pm 0.227$ & 0 \\
\hline$\left\langle\tilde{T}^{\text {AGBS }}\right\rangle$ & 0.974 & $3.601 \pm 0.296$ & $0.181 \pm 0.004$ & $3.771 \pm 0.066$ & $3.959 \pm 0.217$ & 1.224 \\
\hline \hline
\end{tabular}

TABLE I: Results from the fit to the $F_{2}$ data, where values of the parameters with their respective errors are indicated, together with the $\chi^{2}$ per data point.

When the fluctuations are included, some crucial changes take place in the high energy evolution. Different realizations of the target up to rapidity $Y$ generate fronts with different positions $\rho_{s} \equiv \ln Q_{s}^{2}(Y) / k_{0}^{2}$. The single event amplitude is still a traveling front, which, at asymptotic rapidities satisfies geometric scaling, but with a speed $\lambda^{*}$ smaller than the correspondent mean field prediction.

The saturation scale becomes a random variable with average value and dispersion given, respectively, by

$$
\left\langle\rho_{s}\right\rangle=\exp \left[\lambda^{*} Y\right], \quad \sigma^{2}=\left\langle\rho_{s}^{2}\right\rangle-\left\langle\rho_{s}\right\rangle^{2}=D \bar{\alpha} Y .
$$

The probability distribution of $\rho_{s}$ is approximately Gaussian [12]

$$
P_{Y}\left(\rho_{s}\right) \simeq \frac{1}{\sqrt{\pi \sigma^{2}}} \exp \left[-\frac{\left(\rho_{s}-\left\langle\rho_{s}\right\rangle\right)^{2}}{\sigma^{2}}\right]
$$

One must then distinguish the single event amplitude from the average amplitude, given by $\left(\rho \equiv \ln k^{2}\right)$

$$
\left\langle T\left(\rho, \rho_{s}\right)\right\rangle=\int_{-\infty}^{+\infty} d \rho_{s} P_{Y}\left(\rho_{s}\right) T\left(\rho, \rho_{s}\right) .
$$

A crucial consequence of the fluctuations is that, at sufficiently high energies, geometric scaling will not be present in the evolution of the average amplitude, but it will be replaced by a new type of scaling, the diffusive scaling $[11,13]$

$$
\left\langle T\left(\rho, \rho_{s}\right)\right\rangle=\mathcal{T}\left(\frac{\rho-\left\langle\rho_{s}\right\rangle}{\sqrt{D Y}}\right),
$$

where $D$ is the diffusion coefficient.

\section{DIS AND FLUCTUATIONS}

In Ref. [6], the IIM and GBW models were considered in the presence of fluctuations to reproduce the measurements of $F_{2}$ proton structure function from DIS at HERA [14,15]. For each model, the corresponding parametrization was considered as a single-event amplitude, the average (13) was performed and the resulting amplitude replaced into the expression of $F_{2}$, with the diffusion coefficient being taken as a free parameter. violating has gained a large improvement after effects, while in the IIM model these improvement was not so large, due explicitly violates the geometric scaling.

In these proceedings, we propose the same investigation done in [6], not in coordinate space, but in momentum space through AGBS model. In this analysis, all the last HERA data measurements of the proton structure function from $\mathrm{H} 1 \mathrm{Col}-$ laboration [14] and ZEUS Collaboration [15] are fitted. The kinematical range is given by

$$
\begin{gathered}
x \leq 0.01 \\
0.045 \leq Q^{2} \leq 150 \mathrm{GeV}^{2}
\end{gathered}
$$

which gives a total of 279 data points. The first limit means that we are interested in the high-energy regime of DIS and the second one is low enough to allow an investigation without considering DGLAP corrections. The above range is larger than the one used in [6], then it seems the present analyis is more precise.

Concerning the parameters, we keep fixed $\bar{\alpha}=0.2$, which enters into the amplitude through Eq.(7), $\gamma_{c}=0.6275$, whose value corresponds to the one obtained from the LO BFKL kernel, and $D=1.224$, which is the value found numerically by solving the $(1+1)$ dimensional toy model [16]. The other parameters in the amplitude, $\lambda, k_{0} 2$ and $\chi^{\prime \prime}$ are left to be free, as well as the proton radius $R_{p}$, which fixes the normalisation of the dipole-proton cross section with respect to the dipoleproton amplitude. This analysis includes light quarks only and the value used for their masses is $m_{u, d, s}=50 \mathrm{MeV}$.

The results of the fit to the $F_{2}$ structure function are shown in Table I, where the parameters and the $\chi 2 /$ n.o.p. (this notation means $\chi^{2}$ per number of points) are presented. In this table we compare the results obtained using the average and the single-event amplitudes. Fig. 1 shows $F_{2}$ as a function of $Q^{2}$ for different values of the rapidity $Y$. One can see that the data are very well fitted by the correspondent curves, as provided by the value of the $\chi 2$ obtained by using the average amplitude.

The value for the $\chi^{2}$ obtained with the average amplitude is not significantly improved with respect to the case with the single event amplitude. The same happens for the parameters which differ from one analysis to another, but also not significantly, except the saturation exponent $\lambda$, which is smaller when the fluctuations are included, in agreement with theoretical predictions. This seems to indicate that, although the fluctuations may be present, they are not so important in the energies at HERA, and more, the scaling violations come mainly from the diffusion term present in the scattering amplitude in the dilute regime (6), which is a mean field (BK) asymptotic prediction for the amplitude.

In order to perform a deeper analysis, one can fix different values for the diffusion coefficient $D$ and, of course, to fit the data by lefting $D$ as a free parameter. This is the goal our next work [17]. 


\section{$F_{2}$ Structure Function}

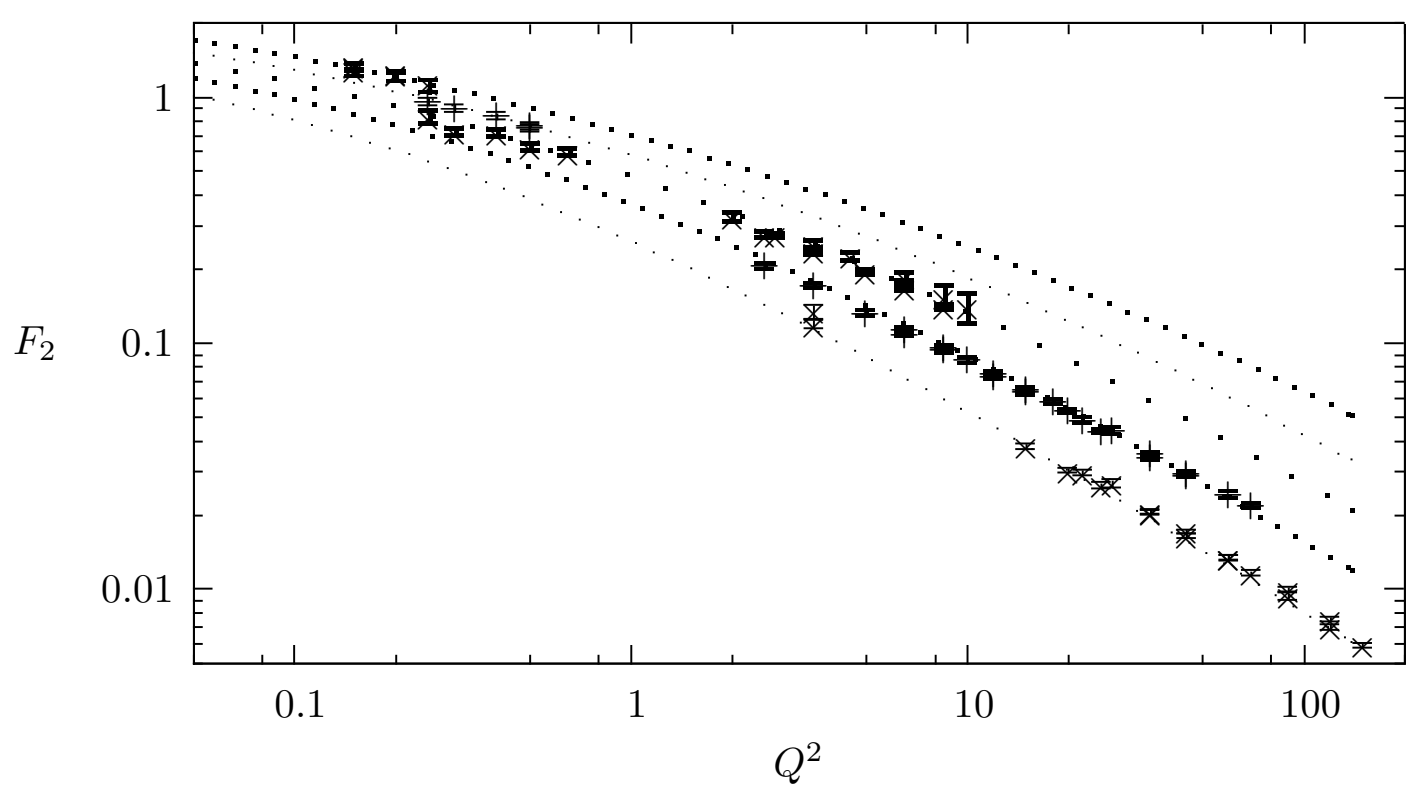

FIG. 1: The $F_{2}$ proton structure function as a function of $Q^{2}$ for different values of rapidity $Y$. From bottom to top, $Y=0,2,4,6,8$.

[1] A. H. Mueller, Nucl. Phys. B 415, 373 (1994); A. H. Mueller and B. Patel, Nucl. Phys. B 425, 471 (1994); A. H. Mueller, Nucl. Phys. B 437, 107 (1995).

[2] I. Balitsky, Nucl. Phys. B 463, 99 (1996) [arXiv:hep$\mathrm{ph} / 9509348]$

[3] J. Jalilian-Marian, A. Kovner, A. Leonidov, and H. Weigert, Nucl. Phys. B 504, 415 (1997) [arXiv:hep-ph/9701284]; E. Iancu, A. Leonidov, and L. D. McLerran, Nucl. Phys. A 692 583 (2001) [arXiv:hep-ph/0011241]; H. Weigert, Nucl. Phys. A 703, 823 (2002) [arXiv:hep-ph/0004044].

[4] Y. V. Kovchegov, Phys. Rev. D 60, 034008 (1999) [arXiv:hepph/9901281]; Phys. Rev. D 61, 074018 (2000) [arXiv:hep$\mathrm{ph} / 9905214]$.

[5] S. Munier and R. Peschanski, Phys. Rev. Lett. 91, 232001 (2003); Phys. Rev. D 69, 034008 (2004); Phys. Rev. D 70, 077503 (2004).

[6] M. Kozlov, A. Shoshi, and W. Xiang, JHEP 0710, 020 (2007) [arXiv:0707.4142 [hep-ph]].

[7] K. Golec-Biernat and M. Wusthoff, Phys. Rev. D 59, 014017 (1999) [arXiv:hep-ph/9807513].

[8] E. Iancu, K. Itakura, and S. Munier, Phys. Lett. B 590, 199 (2004) [arXiv:hep-ph/0310338].

[9] J. T. de Santana Amaral, M. B. Gay Ducati, M. A. Betemps, and G. Soyez, Phys. Rev. D 76, 094018 (2007) [arXiv:hep- $\mathrm{ph} / 0612091]$.

[10] L. N. Lipatov, Sov. J. Nucl. Phys. 23, 338 (1976); E. A. Kuraev, L. N. Lipatov, and V. S. Fadin, Sov. Phys. JETP 45, 199 (1977); I. I. Balitsky and L. N. Lipatov, Sov. J. Nucl. Phys. 28, 822 (1978).

[11] E. Iancu and D.N. Triantafyllopoulos, Nucl. Phys. A 756, 419 (2005).

[12] C. Marquet, G. Soyez, and B. W. Xiao, Phys. Lett. B 639, 635 (2006).

[13] E. Iancu and A.H. Mueller, Nucl. Phys. A 730, 494 (2004); A. H. Mueller and A.I. Shoshi, Nucl. Phys. B 692, 175 (2004); Y. Hatta, E. Iancu, C. Marquet, G. Soyez, and D. N. Triantafyllopoulos, Nucl. Phys. A 773, 95 (2006).

[14] C. Adloff et al. [H1 Collaboration], Eur. Phys. J. C 21, 33 (2001) [arXiv:hep-ex/0012053].

[15] J. Breitweg et al. [ZEUS Collaboration], Phys. Lett. B 487, 273 (2000) [arXiv:hep-ex/0006013]; S. Chekanov et al. [ZEUS Collaboration], Eur. Phys. J. C 21, 443 (2001) [arXiv:hepex/0105090].

[16] E. Iancu, J. T. de Santana Amaral, G. Soyez, and D. N. Triantafyllopoulos, Nucl. Phys. A 786, 131 (2007).

[17] E. Basso, M. B. Gay Ducati, E. G. de Oliveira, and J. T. de Santana Amaral, in preparation. 